\title{
Is one swab enough to detect chlamydial infection of the cervix?
}

\author{
P E MUNDAY, $+\mathrm{J}$ M CARDER, * N F HANNA, + AND D TAYLOR-ROBINSON* \\ From the *Division of Sexually Transmitted Diseases, MRC Clinical Research Centre, Harrow, Middlesex \\ and the †Praed Street Clinic, St Mary's Hospital, London
}

SUMMARY Three swabs were taken from the cervix of each of 104 women for the detection of Chlamydia trachomatis. The processing of three swabs instead of one increased the isolation rate by only $2 \%$, and later swabs did not result in the production of more chlamydial inclusions than first swabs. In most clinics, therefore, a single cervical swab is adequate to detect chlamydial infection.

\section{Introduction}

Chlamydia trachomatis is an important aetiological agent in disease of the genital tract in men and women. ${ }^{1}$ Clinical and epidemiological studies were facilitated by the development of a tissue culture technique for isolation, ${ }^{2}$ which superseded the use of embryonated eggs. Although many modifications of the original method have been described, ${ }^{3}$ none has been shown to produce a noticeable increase in sensitivity. Furthermore, serological studies, repeat attempts to isolate the micro-organism from patients treated with placebo, and examination of sexual partners, all suggest that failure to detect chlamydiae using standard isolation procedures is rare. ${ }^{4}$ Nevertheless, the possibility exists that isolation techniques might not yet be optimal and that patients apparently regarded as chlamydia negative might, in fact, harbour the micro-organism.

One aspect of assessing the sensitivity of an isolation technique is the enumeration of chlamydiae recovered from multiple specimens taken from the same site at the same time. ${ }^{5}$ The results of some studies $^{6}$ (and Dunlop et al, unpublished observation) have suggested that more than one specimen should be processed in order to obtain a maximum chlamydial isolation rate. If an increased isolation rate occurs because the first swab sometimes fails to detect chlamydiae in mucus or debris whereas later swabs remove epithelial cells which are essential for the isolation of chlamydiae (a "sequence" effect), a

Address for reprints: $\operatorname{Dr} P$ E Munday, Division of Sexually Transmitted Diseases, MRC Clinical Research Centres, Watford Road, Harrow, Middlesex HA1 3UJ

Accepted for publication 4 March 1984 simple procedure to increase the isolation rate at no extra cost would be to discard the first swabs and process the later ones. Alternatively, if an increased isolation rate occurs because positive results obtained from later swabs are added to those obtained by the first, even though the isolation rate for each swab in the sequence is about the same (a "chance" effect related to the inherent failure rate of any method), it would be necessary to process all specimens to obtain the maximum isolation rate.

If increasing numbers of chlamydiae, reflected in increasing numbers of inclusions, were found in sequential specimens, it would strengthen the argument in favour of a sequence rather than a chance effect. We therefore conducted a quantitative study to distinguish between these two possibilities.

\section{Method}

Three sequential specimens were taken from 104 women who attended sexually transmitted disease clinics because they were sexual partners of men with gonorrhoea or non-gonococcal urethritis. Each specimen was taken with a polyester sponge swab, ${ }^{7}$ which was rotated in the cervix and then expressed in $0.8 \mathrm{ml}$ sucrose phosphate transport medium (2SP) containing $10 \%$ fetal calf serum, vancomycin, streptomycin, and nystatin. The vials of medium were immediately snap frozen in liquid nitrogen for transport to and storage in the laboratory.

C trachomatis was isolated in McCoy cells treated with cycloheximide, which were stained with Giemsa reagent and examined by dark field microscopy. ${ }^{8}$ The number of inclusion forming units in a specimen was calculated by adding the number of inclusions counted in both the cell monolayers, and was expressed on a $\log _{10}$ scale. 


\section{Results}

\section{ISOLATION RATES}

For $99(95 \%)$ of the 104 women the results for each of the three specimens were the same, 34 giving positive and 65 negative results for chlamydiae. Table I shows the results of testing specimens from the five remaining patients. Overall, only two more patients were recorded as chlamydia positive as a consequence of taking second and third specimens. The isolation rate would have been $35.6 \%$ if only one specimen had been taken, $36 \cdot 5 \%$ for two, and $37.5 \%$ for three specimens. The isolation rates for the first, second, and third swabs alone were $35 \cdot 6 \%$, $33 \cdot 7 \%$, and $36 \cdot 5 \%$ respectively.

TABLE I Chlamydial isolation from five patients whose sequential samples provided inconsistent results

\begin{tabular}{llll}
\hline & \multicolumn{4}{l}{$\begin{array}{l}\text { Chlamydial isolation from swabs taken in the } \\
\text { following sequence: }\end{array}$} \\
$\begin{array}{llll}\text { Case } \\
\text { No }\end{array}$ & First & Second & Third \\
\hline 1 & - & + & + \\
2 & + & - & + \\
3 & + & - & + \\
4 & - & - & + \\
5 & + & &
\end{tabular}

\section{NUMBERS OF INCLUSIONS}

Table II shows the numbers of chlamydial inclusions produced by the first specimens compared with the numbers produced by the third specimens. In most cases, the numbers of inclusions produced in tests on the first and third swabs were the same. Where there was a discrepancy between the results of tests on the first and third swabs, the chance of the first swabs producing a larger or smaller number of inclusions than the third swabs was almost equal to that of the third swabs producing a larger or smaller number than the first swabs. The distribution of the numbers of inclusions seen when those produced by the first and second specimens were compared, and when those produced by second and third specimens were compared, was similar to the distribution seen in table II.
When all three specimens were considered, the number of inclusions produced by each specimen was the same in 17 cases, there being more inclusions produced by the first specimen than the third in six cases, and more inclusions by the third specimen than the first in seven cases. In a further four cases the numbers of inclusions produced by the first and third specimens were the same but more (in two cases) or less (in two cases) resulted from the second specimen. Thus there was no consistent trend of increasing or decreasing numbers of inclusions when sequential samples were taken.

\section{Discussion}

The results of this study indicated that isolation of $C$ trachomatis in McCoy cells treated with cycloheximide is a sensitive technique in our hands. If the procedure were only on the threshold of detecting chlamydiae it would be expected that the isolation rates for the first, second, and third specimens would be quite dissimilar and that the numbers of inclusions counted for each set of specimens would also be dissimilar. This was not the case as there was no "sequence" effect, although the technique has a small failure rate, like most biological tests, which could be reduced by taking multiple swabs. We could not, however, justify gaining a slightly increased isolation rate as it would be offset by a greatly increased cost.

These results are compatible with those of Schofield who found that $13(7 \%)$ of 180 women had positive results for chlamydiae only from a second swab. ${ }^{6}$ It is not possible, however, to assess the sensitivity of the procedure used, as the proportion of patients who had a positive first specimen and a negative second specimen was not presented. For the same reason, it is not possible to judge the sensitivity of the procedure used by Dunlop et al (unpublished observation), who obtained positive results in $73 \%$ of women with a first swab, an additional $17 \%$ with a second swab, and $10 \%$ with a third. Their results could be regarded as compatible with ours if their method was less sensitive than ours.

TABLE II Comparison of the numbers of chlamydial inclusions detected in tests on first and third specimens from 104 women

\begin{tabular}{|c|c|c|c|c|c|c|c|}
\hline & & \multicolumn{6}{|c|}{ No of patients whose first swab produced the indicated No of inclusions } \\
\hline & & 0 & $<10^{1}$ & $>10^{1}<10^{2}$ & $>10^{2}<10^{3}$ & $>10^{3}<10^{4}$ & $\geqslant 10^{4}$ \\
\hline
\end{tabular}


The overall sensitivity of the isolation procedure in the study reported by Embil et al must also be questioned. ${ }^{9}$ They took five specimens from the cervix of each patient, and chlamydiae were shown in one or more specimens from 61 women, a greater chlamydial isolation rate being found for fourth and fifth specimens than for first and second ones. However, failure to isolate from the second, fourth, and fifth specimens of 14 , eight, and five patients respectively, whose first specimens gave positive results suggests a lack of sensitivity of the isolation procedure, which may have contributed to the benefit seen from taking multiple samples.

The results of this study indicate that a single specimen is adequate to diagnose chlamydial infection of the cervix if the isolation technique used is sensitive. If, however, clinical suspicion of infection remains despite negative results of chlamydial isolation, the test should be repeated as spurious failures do occur occasionally.

We thank Drs J R W Harris, D Goldmeier, and J Nabarro for access to patients under their care. We also thank Dr A Dyson and Mrs P Clarkson for clinical help, Dr V Bampoe and Miss S Lee for technical help, and Mr D G Altman for statistical advice.

\section{References}

1. Taylor-Robinson D, Thomas BJ. The role of Chlamydia trachomatis in genital-tract and associated diseases. $J$ Clin Pathol 1980; 33:205-33.

2. Gordon FB, Quan AL. Isolation of the trachoma agent in cell culture. Proc Soc Exp Biol Med 1965;118:354-9.

3. Evans RT, Woodland RM. Detection of chlamydiae. Br Med Bull 1983;39: 181-6.

4. Bowie WR, Wang S-P, Alexander ER, Holmes KK. Etiology of nongonococcal urethritis. In: Hobson D,.Holmes KK, eds. Nongonococcal urethritis and related infections. Washington DC: American Society for Microbiology, 1977; 19-29.

5. Wentworth BB. Sensitivity of cell culture for isolation of Chlamydia trachomatis from genital sources. In: Hobson D Holmes KK, eds. Nongonococcal urethritis and related infections. Washington DC: American Society for Microbiology, 1977; 299-303.

6. Schofield CBS. The treatment of chlamydial cervicitis. British Journal of Sexual Medicine 1982; 9:39-42.

7. Oates JK, Selwyn S, Breach MR. Polyester sponge swabs to facilitate examination for genital infection in women. $\mathrm{Br} J$ Vener Dis 1971;47:289-92.

8. Munday PE, Johnson AP, Thomas BJ, Taylor-Robinson D. A comparison of the sensitivity of immunofluorescence and Giemsa for staining Chlamydia trachomatis inclusions in cycloheximide-treated McCoy cells. J Clin Pathol 1980;33:177-9.

9. Embil JA, Thiébaux J, Manuel FR, Pereira LH, MacDonald SW. Sequential cervical specimens and the isolation of Chlamydia trachomatis: factors affecting detection. Sex Transm Dis 1983; 10:62-6. 